\title{
Message from the Editors-in-Chief
}

\author{
James Joshi ${ }^{1}$, Calton $\mathrm{Pu}^{2}$ \\ ${ }^{1}$ University of Pittsburgh, Pittsburgh, USA \\ ${ }^{2}$ Georgia Institute of Technology, Atlanta, USA
}

Received on 24 September 2014, published on 20 October 2014

Copyright $@ 2014$ James Joshi and Calton Pu, licensed to ICST. This is an open access article distributed under the terms of the Creative Commons Attribution licence (http://creativecommons.org/licenses/by/3.0/), which permits unlimited use, distribution and reproduction in any medium so long as the original work is properly cited.

\section{doi: 10.4108/cc.1.2.e1}

We are very pleased to welcome you all to the second issue of this journal. In this issue, we present six selected invited papers that span various aspects of collaborative computing and technologies. In particular, the themes of the papers cover Crowdsourcing, a collaborative approach to secure and oblivious storage, a virtual network infrastructure to enable collaboration, collaborative social navigation support for way-finding for people with disabilities, a collaborative approach to sharing information in social networks, and an approach to understand collaboration and coordination among members in a community. This issue, thus, aims to highlight the role of collaboration technologies and techniques in highly diverse application domains. We thank the authors for their valuable contributions.

In "Reconciling Schema Matching Networks Through Crowdsourcing," authors Viet Hung, Thanh Tam, Miklós and Aberer propose to use crowdsourcing techniques to address the challenge of schema matching during data integration. The proposed Crowdsourcing technique tries to validate the correspondences between the attributes of database schema generated by a large group of people who are non-experts. The work assumes that there is a need to have attribute correspondences established not just between two schemas but also in a network. However, there is a potentially inherent unreliability issue with regards to the users' inputs in Crowdsourcing. The authors propose using integrity and consistency constraints to improve the process. They show that such constraints improve aggregated answers, and also help estimate better the quality of answers of each worker and detect potential spammers.

Frikken, Wang and Atallah in "A Scheme for Collaboratively Processing Nearest Neighbor Queries in Oblivious Storage" address the issue related to confidentiality of both the outsourced data as well as the patterns of access to that data. They propose an extension of the existing Oblivious Storage semantics to include an oblivious index. The proposed oblivious index supports finding the keys nearest to the query in the key-value store. The authors show that the extension has performance that is similar to the existing Oblivious Storage schemes in terms of storage at both clients and servers, as well as the rounds of communication involved.

Juste, Jeong, Eom, Baker and Figueiredo in "TinCan: User-Defined P2P Virtual Network Overlays for Ad-hoc Collaboration," propose a virtual network called TinCan that is based on peer-to-peer private tunnels. Such virtual private networks are important for collaborative environments to provide private, authenticated communication between collaborating partners that belong to multiple institutions and are potentially mobile. The paper presents the architecture and design of TinCan which goes beyond the traditional centralized VPN based approaches. They also present a prototype that supports Windows, Linux, and Android mobile devices, and its experimental evaluation.

In "Wayfinding and Navigation for People with Disabilities Using Social Navigation Networks," authors Karimi, Dias, Pearlman and Zimmerman focus on issues related to safe and independent mobility associated with navigation of unfamiliar indoor as well as outdoor environments. They argue that despite great advances in technologies, the existing solutions for way-finding and navigation do not appropriately address the needs of the people with disabilities (PWDs). The authors further argue that such limitations exist because there is a lack of experience centric approach integrated into these systems and services. They propose using a hybrid approach where the compute-centric and experience-centric methods are combined to overcome such shortcomings to support the needs of the PWDs.

In the next paper titled "SocialCloudShare: a Facebook Application for a Relationship-based Information Sharing in the Cloud," Albertini, Carminati and Ferrari propose an architectural framework for Online Social Networks which uses an application called SocialCloudShare, a social application registered in Facebook, that helps users to move their personal data (e.g., relationships, resources) outside the OSN realm and to store them in the public Cloud. Such an approach has been motivated by the fact that the centralized 
storage of such data can be exploited or misused through use of data mining and marketing analysis tools. The authors' proposed work aims at providing users the security and privacy guarantees over their data by encrypting the resources and by anonymizing their social graphs. Their proposed framework enforces Relationship-Based Access Control (ReBAC) rules over the anonymized social graph, and provides a mechanism to selectively share information and resources.

In the last paper titled "Tracing Coordination and Cooperation Structures via Semantic Burst Detection," authors Lin, Margolin and Lazer explore forms of knowledge distribution structures, such as, coordination and cooperation structures, that are critical for supporting collaboration among the members of a community. The authors propose a method for detecting coordination of strategic communication among the members of various political communities. Key to their proposed work is a sudden burst of use of linguistic constructions, referred to as "rapid semantic convergence," which can be used to signify an existence of coordination. Based on this, the authors analyze the coordination and cooperation networks among the political members using their public statements. Their results show that the networks detected through such semantic bursts reflect underlying tendencies in the social relationships among Senators and exposes some differences in the way different parties achieve coordination in communication.

We strongly believe the readers will find these papers very interesting and useful, and we hope that they will enjoy these papers.

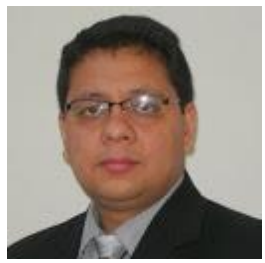

James Joshi (University of Pittsburgh, USA) was born in Nepal. He is an associate professor in the School of Information Sciences at the University of Pittsburgh. He is a co-founder and the Director of the Laboratory of Education and Research on Security Assured Information Systems (LERSAIS), which is NSA and DHS designated National Center of Academic Excellence in Information Assurance Education. He received his MS in Computer Science and $\mathrm{PhD}$ in Computer Engineering degrees from Purdue University in 1998 and 2003. His research interests include Security and Privacy of Distributed Systems, and Trust Management and Information Survivability. He is a recipient of the NSFCAREER award in 2006. His current research activities include advanced access control models, Security and Privacy issues in Cloud Computing and Collaborative systems, Social networks, and Intrusion detection/prevention systems. He served as Program Co-Chair for the IEEE IRI, the International Workshop on Information Assurance, and the International Workshop on Trusted Collaboration, IEEE/ICST CollaborateCom, COMPSAC2009 (as track chair) and ACM SACMAT2009. He has served as General chair of CollaborateCom (multiple times), SACMAT2010.
He currently serves as a Steering committee member of CollaborateCom (co-chair) and SACMAT. He also served as an editorial board member of the International Journals of E-Business research, Network Security and Multimedia and Ubiquitous Engineering. He has served as a program committee member in many international conferences (over 70). He serves in the editorial review board of the International Journal of E-Business Research and the International Journal of Network Security. He is a co-editor of the book titled "Information Assurance: Dependability and Security of Networked Systems".

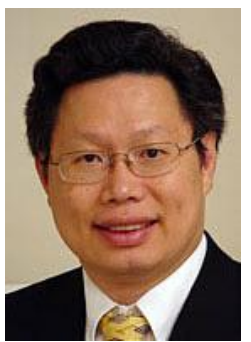

Calton Pu (Georgia Institute of Technologies, USA) was born in Taiwan and grew up in Brazil. He received his $\mathrm{PhD}$ from University of Washington in 1986 and served on the faculty of Columbia University and Oregon Graduate Institute. Currently, he is holding the position of Professor and John P. Imlay, Jr. Chair in Software at the College of Computing, Georgia Institute of Technology. He is leading the Infosphere project, building software tools to support information flow-driven applications such as digital libraries and electronic commerce. Infosphere builds on his previous and ongoing research interests. First, he has been working on next-generation operating system kernels to achieve high performance, adaptiveness, security, and modularity, using program specialization, software feedback, and domain-specific languages. This area has included projects such as Synthetix, Immunix, Microlanguages, and Microfeedback, applied to distributed multimedia and system survivability. Second, he has been working on new data and transaction management by extending database technology. This area has included projects such as Epsilon Serializability, Reflective Transaction Framework, and Continual Queries over the Internet. His collaborations include applications of these techniques in scientific research on macromolecular structure data, weather data, and environmental data, as well as in industrial settings. He has published more than 60 journal papers and book chapters, 150 conferences and refereed workshop papers, and served on more than 90 program committees, including the co-PC chairs of SRDS'95, ICDE'99, CoopIS'02, SRDS'03, DOA'07, and cogeneral chair of ICDE'97, CIKM'01, ICDE'06, DEPSA'07, CEAS'07. 